\title{
Computational problems in introductory physics: Lessons from a bead on a wire
}

\author{
Thomas J. Bensky and Matthew J. Moelter
}

\begin{abstract}
We have found that incorporating computer programming into introductory physics requires problems suited for numerical treatment while still maintaining ties with the analytical themes in a typical introductory-level university physics course. In this paper, we discuss a numerical adaptation of a system commonly encountered in the introductory physics curriculum: the dynamics of an object constrained to move along a curved path. A numerical analysis of this problem that includes a computer animation can provide many insights and pedagogical avenues not possible with the usual analytical treatment. We present two approaches for computing the instantaneous kinematic variables of an object constrained to move along a path described by a mathematical function. The first is a pedagogical approach, appropriate for introductory students in the calculus-based sequence. The second is a more generalized approach, suitable for simulations of more complex scenarios.
\end{abstract}

\section{INTRODUCTION}

In an attempt to invigorate and modernize our introductory calculus-based physics course, we have chosen to include assignments that necessitate the creation of computer animations by the students, ${ }^{1-3}$ with an emphasis on simple computer programming. ${ }^{4}$ Our approach uses computer animation techniques discussed previously. ${ }^{5,6}$ Because our course requires a departmentally determined textbook, ${ }^{7}$ we base our computational curriculum around the discussions and problems found in the text. In creating the curriculum, we found that subtle aspects of many textbook-themed lessons can become problematic when using a computational (i.e., numerical) approach.

As an example, consider the task of developing a computer animation of an object moving along an incline, as in $\mathrm{Knight}^{7}$ page 65. Even such a "simple" system is computationally problematic. The inclined plane is typically presented as a "wedged" surface resting on a flat "ground." As shown in Fig. 1(a), there is a discontinuity where the flat and inclined surfaces meet that is difficult to treat numerically. Student attempts at animating an object moving from the horizontal segment onto the incline will result in unrealistic motion and a failure to illustrate conservation of energy. Altering this problem to replace the discontinuity with a smooth curve, as in Fig. 1(b), is now difficult to treat analytically as the slope is no longer constant. One will likely encounter other difficulties when attempting to create computer animations from standard introductory, textbook-style scenarios. ${ }^{8}$

As we know, the main task when studying mechanics is to identify the interactions of an object with its surroundings and to then use Newton's laws to solve for the object's subsequent trajectory $\vec{r}(t)$. However, in the context of an object on a sloped surface, just as students become accomplished at drawing free-body diagrams and applying Newton's laws, we come across a problem such as a snow sled on a frictionless, undulating hill. The alert student will realize that the normal force, and hence the acceleration, will change throughout the sled's motion. Unfortunately, it is difficult to handle such problems analytically and we typically sidestep the issue by introducing energy ideas and give up entirely on solving for the motion of the sled (i.e., its trajectory).

In this paper, we present two approaches to modeling such a system - a "bead" constrained to move on an arbitrarily shaped "wire" (in two dimensions). The first approach is purely pedagogical, set in the context of possible assignments for students in an introductory physics course that includes a computational element. The second approach provides a more robust solution and allows for more complicated curves. We begin with a wire (curve) described by a single-valued function, in the second approach we parameterize the curve so we can handle multi-valued functions. Results for both approaches are presented.

\section{NEWTON'S LAWS FOR AN OBJECT CONSTRAINED TO A CURVE}

Consider an object of mass $m$ constrained to move on a frictionless wire in a plane, as shown in Fig. 2. For now, the wire can be any smooth shape characterized by a single-valued function $y=f(x)$. Our goal is to obtain complete knowledge of the kinematic variables for a computer-animated investigation. It is tempting to borrow standard techniques (e.g., a rotated coordinate system, etc.) to approach this problem. However, the normal force will change in both magnitude and direction, so this is not an appropriate way to proceed.

Advanced texts on mechanics ${ }^{9,10}$ treat this problem-a "bead on a wire" — using either a Hamiltonian or Lagrangian analysis. Either method leads to one or more differential equations in the generalized position variable that can usually only be solved numerically. ${ }^{11}$ The complexity of this technique in yielding the trajectory of the object is apparent even at the advanced level, as the typically non-linear differential equation is normally simplified to find either general features of the motion (e.g., extrema) or to find positions about which small oscillations can occur. This is less than ideal from an introductory mechanics perspective because it loses sight of our core pedagogical goal: if one can find an object's acceleration, then its trajectory follows from the basic kinematic equations of introductory physics. ${ }^{4}$ 


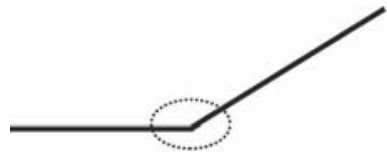

(A)
(B)

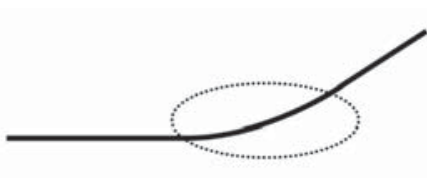

Fig. 1. Examples of the difficulties with a path having a flat-to-incline transition, as indicated in the dotted region. In (A) there is a discontinuity in the curve, which is difficult to treat numerically. In (B) the transition is smoother but there is a non-constant slope, which is difficult to treat analytically.

For a computer adaptation, given our pedagogical setting, a standard coordinate system with the $y$-axis anti-parallel to the weight $m \vec{g}$, is chosen. In general, this coordinate system is not parallel to the instantaneous direction of the normal force $\vec{N}$ of the wire on the mass; however, it is student friendly in the sense that it is aligned with the edges of the screen. For the object shown in Fig. 2, we then apply Newton's second law to get

$$
\sum F_{x}=N_{x}=-N \sin \theta=m a_{x}
$$

and

$$
\sum F_{y}=N_{y}-m g=N \cos \theta-m g=m a_{y},
$$

remembering that in general $\theta=\theta(x)$. We seek the components of the acceleration, but only have two equations for the three unknowns $N, a_{x}$, and $a_{y}$. In the standard approach, with judicious choice of rotated axes, there are situations in which one of the components of the acceleration may vanish, but neither component vanishes in this case.

We instead obtain $a_{x}$ in terms of $a_{y}$ by eliminating $N$ from Eqs. (1) and (2), giving

$$
a_{x}=-\left(a_{y}+g\right) \tan \theta=-\left(a_{y}+g\right) y^{\prime},
$$

where $\tan \theta=d y / d x$ is the slope of the wire and we use a prime to denote derivatives with respect to $x$. At this point, we can go no further because we have one equation with two unknowns $\left(a_{x}\right.$ and $\left.a_{y}\right)$. To make further progress, we make use of the constraint that the object must remain on the wire.

\section{EFFECTS OF THE CONSTRAINT: POSITION, VELOCITY, AND ACCELERATION}

The object is constrained to travel along a wire with a shape given by $y=f(x)$, where $f(x)$ is some continuous, differentiable function. This constraint dictates that the components of position, velocity, and acceleration are not independent and can therefore be expressed in terms of each other. Differentiating with respect to time $t$ leads to

$$
v_{y}=\frac{d y}{d t}=\frac{d y}{d x} \frac{d x}{d t}=y^{\prime} v_{x}
$$

and

$$
a_{y}=y^{\prime \prime} v_{x}^{2}+y^{\prime} a_{x}
$$

where we have employed the chain rule and used the definitions $v_{x}=d x / d t$ and $a_{x}=d v_{x} / d t$, mathematical steps well

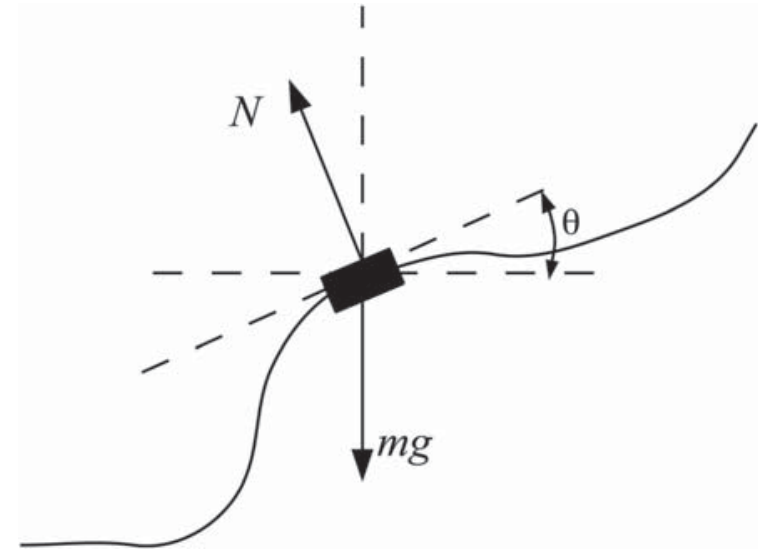

Fig. 2. An object (bead) constrained to an arbitrary curve (shown solid). The normal force $(\vec{N})$ and weight $(m \vec{g})$ are shown. The analytical solution uses a $y$-axis instantaneously parallel to $\vec{N}$. The numerical solution is better found taking the $y$-axis anti-parallel to $m \vec{g}$.

within the capability of students enrolled in a calculus-based physics course. Note that the relationship between $a_{x}$ and $a_{y}$ depends on the shape of the wire (and the constraint that the object remains on the wire) and the horizontal component of velocity $v_{x}$. Substituting Eq. (5) into Eq. (3) then gives

$$
a_{x}=\frac{-\left(y^{\prime \prime} v_{x}^{2}+g\right) y^{\prime}}{1+y^{\prime 2}} \text {. }
$$

The computational adaptability of this result should now be apparent. Given a differentiable function describing the wire, we need its first two derivatives and the speed of the object to determine $a_{x}$ using Eq. (6); we can then find $a_{y}$ from Eq. (5). In principle, these acceleration components may be used to compute the velocity and position of the object. For straight wires, the standard constant-acceleration kinematic equations can be used. If the wire is curved the acceleration components will vary as you move along. In this case we can only use the standard kinematics equations over short time intervals where the acceleration is essentially constant. We emphasize the utility of the approach and this result in the context of introductory physics. The steps are an incremental extension to what students can already do, bringing together introductory calculus and physics in the context of modeling a problem.

We note that Eq. (4) also gives a relationship between $v_{y}$ and $v_{x}$, but for algorithmic consistency in this pedagogical setting we choose a numerical approach to compute $v_{y}$ from $a_{y}$. In this work we also use Eqs. (1) and (2) to compute $N_{x}=m a_{x}$ and $N_{y}=m\left(a_{y}+g\right)$, allowing for an opportunity to study the normal force as the object moves along the wire. Naturally, other quantities are now computable, such as energy, momentum, etc.

Suppose these results are checked against the traditional textbook problem of an object on a fixed incline at an angle of $45^{\circ}$ with respect to the horizontal (slope $\left.=1\right)$. The above results predict that $v_{y}=v_{x}, a_{x}=-g / 2$, and $a_{y}=a_{x}$. To compare to the traditional solution requires that the predicted values of $a_{x}$ and $a_{y}$ are rotated (counterclockwise by $45^{\circ}$ ) into the more familiar analytical coordinate system via

$$
\left(\begin{array}{l}
a_{x}^{\operatorname{trad}} \\
a_{y}^{\operatorname{trad}}
\end{array}\right)=\left(\begin{array}{cc}
\cos 45^{\circ} & \sin 45^{\circ} \\
-\sin 45^{\circ} & \cos 45^{\circ}
\end{array}\right)\left(\begin{array}{l}
-g / 2 \\
-g / 2
\end{array}\right) .
$$




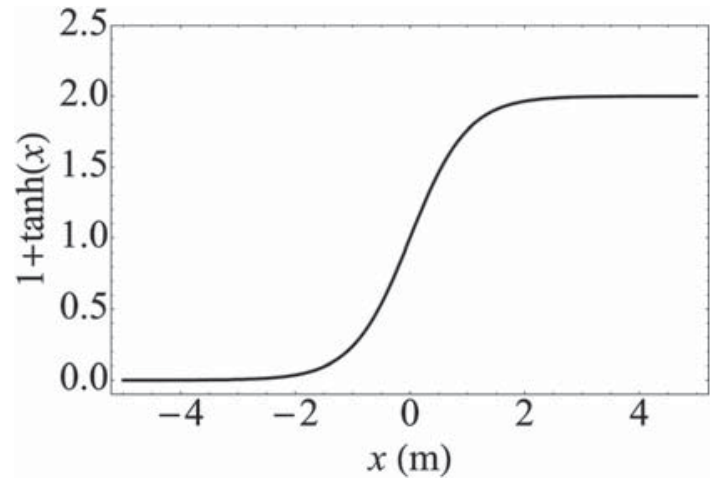

Fig. 3. A plot of the function $1+\tanh (x)$. The two horizontal sections connected by a smooth transition make it a useful candidate for studying an object constrained to a sloped wire.

Such a rotation gives $a_{x}^{\text {trad }}$ and $a_{y}^{\text {trad }}$ in a coordinate system with $y$-axis parallel to the normal force. Expanding Eq. (7) indeed yields $a_{x}^{\text {trad }}=-g \sin 45^{\circ}$ and $a_{y}^{\text {trad }}=0$, the familiar textbook results.

\section{A COMPUTATION-FRIENDLY INCLINED WIRE}

As a reasonable choice for representing an "inclined" wire for computational consideration, we consider the function $y(x)=1+\tanh (x)$ shown in Fig. 3. Its appeal is readily apparent-two horizontal spans smoothly connected to an inclined midsection with "ground level" at $y=0$ and no discontinuities. Given $y(x)$, it is an appropriate exercise for introductory physics students to determine $a_{x}$ and $a_{y}$ by calculating $y^{\prime}(x)$ and $y^{\prime \prime}(x)$ and using Eqs. (5) and (6). We now emphasize that with $a_{x}$ and $a_{y}$ known, simply choosing $v_{x 0}$ and $v_{y 0}$ makes the kinematic equations introduced early in the course the link to the object's trajectory. We can apply these equations provided we use sufficiently small time steps so the acceleration can be considered constant.

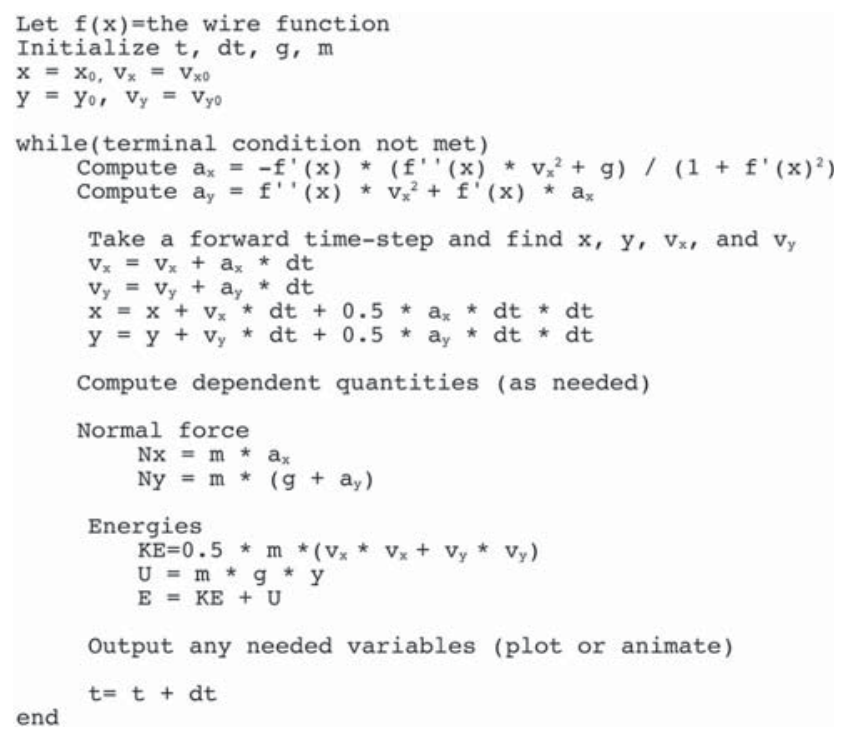

Fig. 4. Pseudocode for simulating the time evolution of an object (of mass $m$ ) constrained to move along the curve given by the function $y=f(x)$. Our experience shows that, similar to the code shown in Ref. 4, this algorithm is quite useable in an introductory physics course.

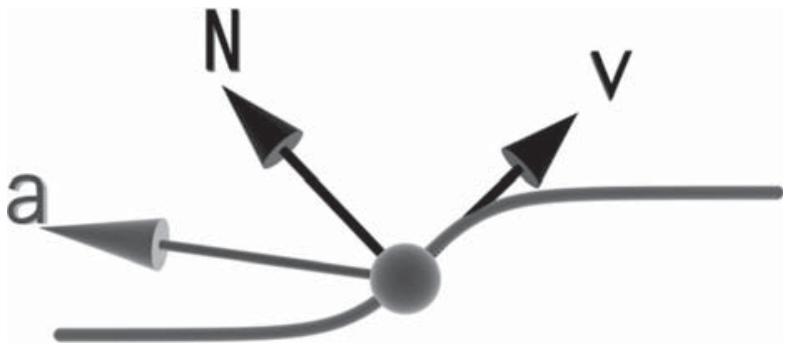

Fig. 5. Instantaneous normal force $(\vec{N})$, velocity $(\vec{v})$, and acceleration $(\vec{a})$ vectors displayed for an object constrained to a wire described by $y(x)=1+\tanh (x)$. These vectors were obtained using $m=0.5 \mathrm{~kg}, g=9.8 \mathrm{~N} /$ $\mathrm{kg}, x_{0}=-5 \mathrm{~m}, y_{0}=0 \mathrm{~m}, v_{x 0}=8 \mathrm{~m} / \mathrm{s}$, and, from Eq. (4), $v_{y 0}=1.8 \times 10^{-4} \mathrm{~m} / \mathrm{s}$ (enhanced online) [URL: http://dx.doi.org/10.1119/1.4773561.1].

In Fig. 4, we give the pseudo code for a computational algorithm used to simulate the behavior of the object (mass $m$ ) on a wire described by the function $y=f(x)$. When implemented, a frame-by-frame animation of the time evolution of the object's trajectory can be created, including the drawing of instantaneous $\vec{v}, \vec{N}$, and $\vec{a}$ vectors, as shown in the single frame of Fig. 5. ${ }^{12}$ Further checks of the method include monitoring kinetic, potential and total energies as the object moves along the wire, as shown in Fig. 6. We note a slight increase in total energy, which is typical and due to the simplistic Euler-steps taken by our numerical model (which we use exclusively in introductory physics). ${ }^{13}$ For each curve chosen, this technique can be used to create unique energy plots like those shown in Fig. 6 that provide challenging pedagogical questions related to the "give and take" between kinetic and potential energies that leave the total energy constant.

While our classroom work has primarily used the animation techniques outlined in Refs. 5 and 6, we support the call for standardization of such computational work ${ }^{14}$ and have also developed an "Easy Java Simulation" version. ${ }^{15}$

\section{INTRODUCTORY PHYSICS PEDAGOGY}

As we conclude our treatment of Newton's laws, the aforementioned theory is used by our students to write short computer programs to animate an object traversing a prescribed path. Our interactions with students in regard to such

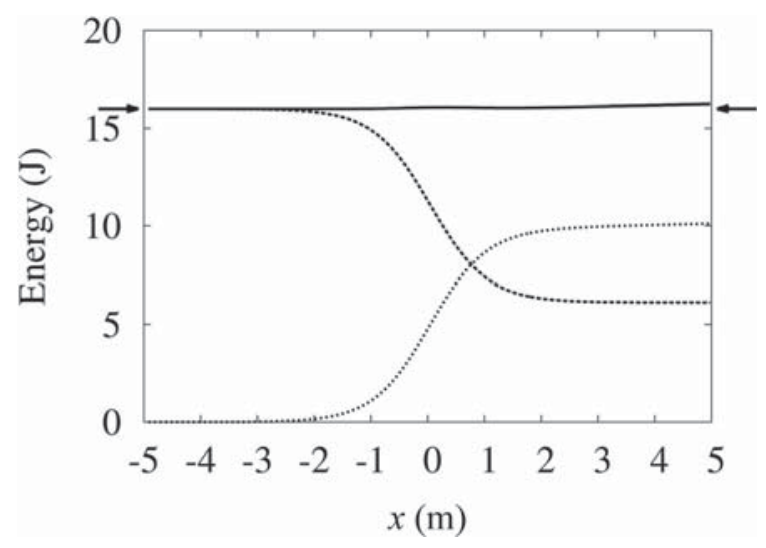

Fig. 6. Total (solid), kinetic (dashed), and potential (dotted) energies as a function of horizontal position for an object on the $1+\tanh (x)$ wire (see Fig. 3). The parameter values are the same as in Fig. 5, with the object traversing the curve from left to right. The arrows indicate $16 \mathrm{~J}$ of initial total mechanical energy. The slight increase in total energy is typical of a numerical integration that uses simple Euler steps. 
work have motivated some new ideas for pedagogy. The first is in the examination of the normal force, which our theory is able to continuously deliver as an object traverses its prescribed path. The second is in the assessment of, and student reaction to, numerical results delivered by the computer. We discuss these below.

\section{A. Examination of the normal force}

With knowledge of the instantaneous kinematic variables provided by the numerical integration, this technique also allows for the determination of the instantaneous components of the normal force using Eqs. (1) and (2). This is an important outcome of this work and in our experience it opens up several pedagogical avenues, even if just used by an instructor as supplementary lecture material.

The components of the normal force naturally lead to the full normal force vector as shown in Fig. 5. In a computer animation (such as that shown in Ref. 12), we find it both instructive and engaging to emphasize the dynamic nature of the normal force as the object moves from left to right along the curve, imagining that the object is a roller coaster car constrained to its track. Figure 7 shows the magnitude and direction of the normal force for the path $1+\tanh (x)$. The angle between the normal vector and the tangent to the wire is instantaneously computed using the dot product between these vectors. As expected, this angle remains at essentially $90^{\circ}$. Also shown in this figure is the exact (solid curve) and numerically calculated (dots) trajectories of the object.

The top plot in Fig. 7 shows the magnitude of the normal force with several intriguing aspects (the vertical lines are guides to the eye). First, the normal force is (approximately) equal to the weight of the car $(\approx 5 \mathrm{~N})$ at positions (a) and (e), where the track is essentially flat. Second, there is a gradual increase in the normal force as the car ascends the track, reaching a maximum at position (b). This readily illustrates the structural demands on the track; it must be able to provide a "reaction force" that is much larger than the weight of the car depending on its travel speed. Third, as the car approaches the top of the track the normal force decreases to zero at position (c), indicating a region where the only force on the car is its weight. Such free-fall (or near free-fall) motion is the cause for the familiar "butterflies in the stomach" one feels at such points in actual motion. Fourth, after passing through zero at point (c), the normal force begins growing in the downward direction, indicating a need for the track to pull down on the object to keep it from flying off of the track. This behavior occurs between positions (c) and (d) and provides another illustration of the structural demands on such a track (and is one reason why its important to have your seatbelt fastened on a roller coaster). After peaking in the downward direction, the normal force again reaches zero at point (d) before leveling off at the weight of the car at position (e). These salient features are all functions of the initial speed of the car.

As complex as the equations in Sec. III may appear to the freshman student, they are simply new mathematical functions that, when evaluated, give the components of an object's acceleration. We have found these equations to give a unique opportunity to emphasize the links between the force on an object, the acceleration, and the trajectory. The depth of the equations adds lesson-plan value, as a mathematical function must first be chosen and worked on with some basic differential calculus before $a_{x}$ and $a_{y}$ can actually be found. Once
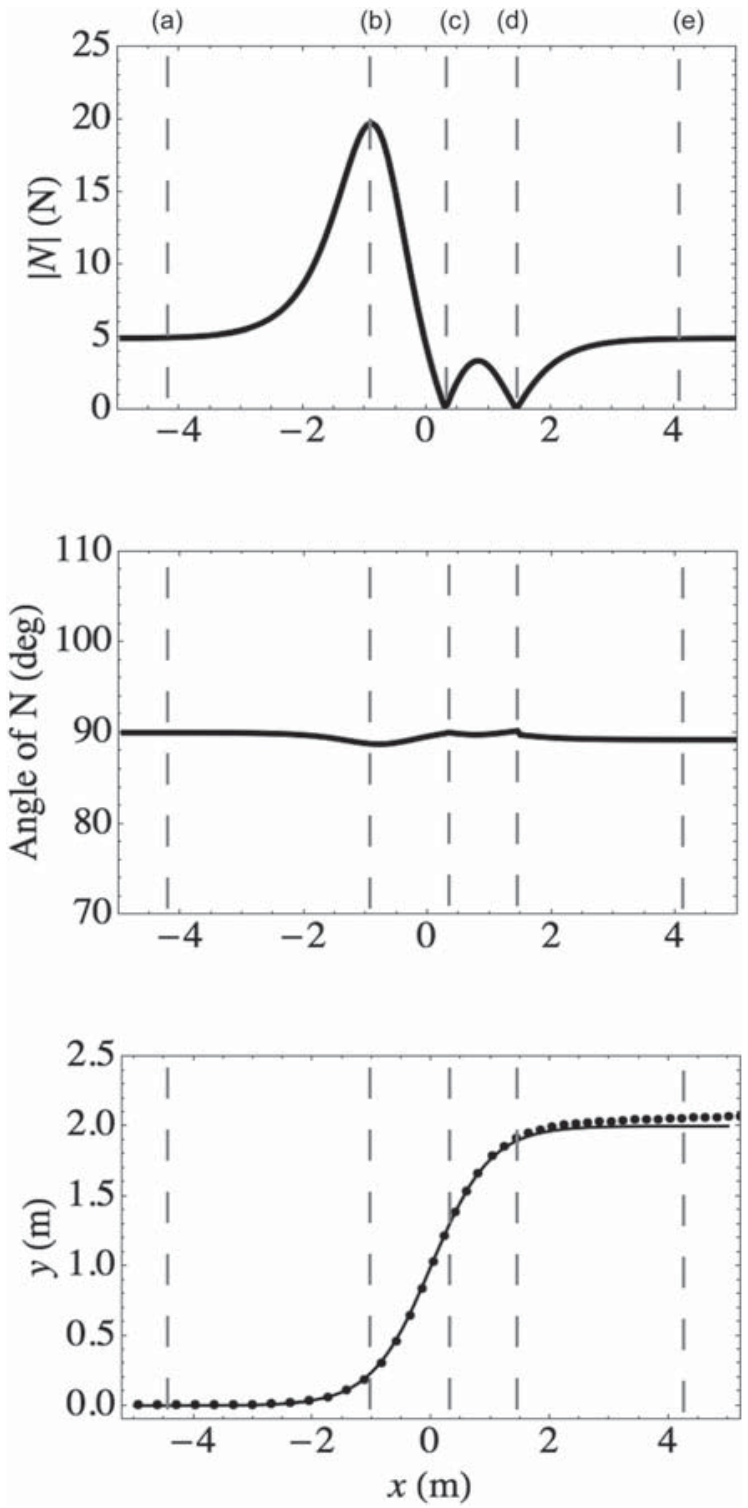

Fig. 7. Summary of the motion of a bead constrained to move along a wire in the shape shown in Fig. 3. The normal force magnitude (top) and direction (center) and the vertical position (bottom) are all plotted as a function of horizontal position. In the bottom plot, the solid curve shows the exact result and the dots are the numerical results. The labelled vertical lines are guides to the eye at interesting pedagogical points (see text). All plots were obtained using the same parameter values as in Fig. 5.

found, the usual kinematic equations can be used to evolve the values of $x$ and $v_{x}$. Moreover, the computational availability of these basic kinematic variables allows for a variety of quantities to be investigated further, of which we have found the normal force and energy to be compelling for the introductory audience. Another example is to note that, while watching the animation of the object on the curved portions of the trajectory, ${ }^{12}$ the acceleration vector points roughly toward the center of curvature of the track between points (c) and (d). This observation allows for a natural connection to uniform circular motion discussed elsewhere in the course.

\section{B. Assessment of and response to numerical results}

Unbeknownst to students at this level, using a computer in the manner described is a new way of solving physics 
problems, and an important issue is that of checking the results. For an analytical homework problem, the students' "gold standard" is the back-of-book answer. For laboratory work, students are encouraged to work with uncertainties. For numerically derived solutions, we have found the assessment of results to be more multifaceted.

When working with animations we do not solely focus attention on a singular result, perhaps at the end of the integration process. Instead, we take the animation itself as part of the solution. Thus, a zeroth-order assessment might be to simply ask "does the animation look right?" We find that a careful examination of the time-evolving vectors and their interplay can often expose implementation errors, which, when corrected, can generate valuable discussions about the underlying physics.

Beyond the zeroth-order assessment, instantaneous variables can be examined at specific times during the animation. If the student is modeling an analytical (textbook) problem, they can compare their numerical results to the exact solution. ${ }^{16}$ We may also consider "constants of the motion" such as energy and momentum, both of which are important concepts in introductory physics. Although we consistently emphasize these points, students are accustomed to the satisfying exactitude of end-of-chapter answer comparisons of their analytical results. Unfortunately, numerical results will generally not lead to perfect agreement and this can be bothersome to the students.

In addressing numerical deviations from the exactness students crave, we must be careful to use an approach that is appropriate for this level of instruction. The practicing physicist knows the usual caveats and proper adaptations of numerical algorithms, but these are not appropriate at this level. Introducing computation into a traditional introductory course is a significant additional cognitive load for the students, and an instructor must take this into consideration.

For the sake of the students, we feel we must stick with the kinematic equations (e.g. $\Delta x=v_{0} \Delta t+a \Delta t^{2} / 2$ ) found in the text. Even simple modifications to this, such as EulerCromer, will raise too many off-topic questions. We also steer clear of simple "predictor-corrector" ideas or other adaptive algorithms. Objections to this self-imposed requirement are a much larger issue in physics pedagogy than the efforts presented in this work. ${ }^{17}$ Choosing to not use these well-known modifications gives us only a single quantity to adjust, the chosen time-step $\Delta t$. However, the ill-suited nature of $a \Delta t^{2} / 2$ to any work beyond analytical textbook solutions means a smaller $\Delta t$ will not always fix numerical errors; in any case, simply choosing smaller and smaller $\Delta t$ 's is not responsible computing. Therefore, we must deal with the inevitable numerical deviations and this has led to stimulating and productive pedagogy for the otherwise moribund introductory mechanics course. We present the following as an example of our discussions.

When comparing analytical and numerical solutions we borrow ideas from the laboratory, where examining the percent error is important. To our mind numerical errors near $5 \%$ are acceptable at this level of instruction as, like the laboratory, the numerical approach is not analytic. As an example, the total energy presented in Figure 6 is off by $2 \%$ (but growing) by the end of the simulation. A smaller time step, or different integration algorithm would alleviate this. ${ }^{13}$ To the introductory student, our discussion of this erroneous energy proceeds as follows (as part of our usual discussion of energy diagrams ${ }^{7}$ ):
Kinematic quantities vary wildly from start to finish in a given problem (imagine a projectile in flight), and let's not forget that we use these same kinematic variables to compute energy. Let's do the following as our code runs: square the magnitude of the velocity, multiply by the mass of the object, then multiply by $1 / 2$ (i.e., compute $m v^{2} / 2$ ). Next, let's compute the product of the object's mass, gravity, and vertical coordinate (i.e., compute $m g y$ ), and then add these two quantities together. The surprise is that despite hundreds of percent changes in the kinematic variables ( $v$ and $y$ ), this final sum, which is just a particular combination of these variables, stays constant to within a few percent. This is witness to conservation of energy. With Fig. 6 at hand we ask, "Will you not agree that energy is trying to stay constant, despite the monumental changes in the quantities from which is it computed?"

Our focus here is not on a poorly chosen integration technique. It is instead on the usefulness and constraining nature of conservation of energy in a new problem-solving situation. References to the size of the time step required to produce better results are appropriate, as is a diluted (pictorial) motivation of how a more advanced algorithm might work.

The same type of discussion is used to discuss the normal force presented in Fig. 7, which students know should always be perpendicular to the surface. In the center plot of Fig. 7, there is an obvious "ripple" in the direction of the normal force during the object's ascent. This ripple deviates from $90^{\circ}$ by a mere $2 \%$, despite wild changes in the normal force magnitude (top) and track orientation (bottom). We again focus on the numerical normal force "trying" to remain at $90^{\circ}$ with respect to the track.

The most visible shortcoming of our integration technique is that the position of the object deviates from that of the track (bottom plot of Fig. 7). In the simulation that generated this figure, the object deviates from the track by at most $3.5 \%$, and the direction of $\vec{v}(x)$ deviates from $y^{\prime}(x)$ by at most $4 \%$, even for a fairly small step size $(0.005 \mathrm{~s})$. Such deviations highlight the multifaceted assessment of computational work at this level-they are all but imperceptible in an animation produced with a level-appropriate theory, but bothersome when looking for exact answers. Nonetheless, our discussion with the students highlights that we are still seeing a rather remarkable result, as follows:

The "track" as shown in this work is nothing but a collection of "dumb" pixels on a computer screen. It is plotted from the $y(x)$ we chose as a visual aid during the animation. There is no actual interaction between the moving object and these pixels. Remember that the position of the object is found by computing its $x$ and $y$ coordinates from $a_{x}$ and $a_{y}$ [Eqs. (5) and (6)] using Newton's laws. The fact that these laws cause the object to follow a $2.0 \mathrm{~m}$ high path to within $0.07 \mathrm{~m}(3.5 \%)$ while maintaining a near $90^{\circ}$ normal force-to-track angle illustrates their continued success, even in this entirely different setting for physics problem solving.

In sum, given the algorithmic constraints needed at the introductory level, assessing the outcome of student-created computational work needs to be carefully aligned with a perspective that is relatively free of advanced ideas of computation. Errors 
must instead be evaluated and weighed relative to a broader pedagogical view. Careful planning can lead to stimulating discussions showing that fundamental ideas of physics are still observed, although perhaps not in a manner as "exact" as that in the textbook. Finally, we must also look to the original goal of a given computational problem. In the case of this work, we wanted to simulate the motion of an object that was forced to negotiate a prescribed path. We are pleased that a theory can be presented that has a solid footing in the introductory course and allows for compelling, student-created animations with rich pedagogical value.

\section{GENERALIZED TREATMENT OF AN OBJECT CONSTRAINED TO A CURVE}

In the treatment above the wire is described by a singlevalued function, which is perhaps the extent of the appropriateness for introductory physics. However, this precludes the natural possibility of a wire that loops back on itself, as in a "loop-the-loop" roller coaster (see Fig. 8). This restriction can be overcome if we consider the more general case where the wire is any curve in the plane that can be parameterized by a variable $u$, so that $x=x(u)$ and $y=y(u)$. We note that $u$ does not necessarily carry any physical significance. Given such a parameterization, the components of velocity are then

$$
v_{x}=\frac{d x}{d t}=\frac{d x}{d u} \frac{d u}{d t}=x^{\prime} \dot{u}
$$

and

$$
v_{y}=\frac{d y}{d t}=\frac{d y}{d u} \frac{d u}{d t}=y^{\prime} \dot{u},
$$

while those for acceleration become

$$
a_{x}=\frac{d^{2} x}{d u^{2}}\left(\frac{d u}{d t}\right)^{2}+\frac{d x}{d u} \frac{d^{2} u}{d t^{2}}=x^{\prime \prime} \dot{u}^{2}+x^{\prime} \ddot{u}
$$

and

$$
a_{y}=\frac{d^{2} y}{d u^{2}}\left(\frac{d u}{d t}\right)^{2}+\frac{d y}{d u} \frac{d^{2} u}{d t^{2}}=y^{\prime \prime} \dot{u}^{2}+y^{\prime} \ddot{u} .
$$

Here, dots denote differentiation with respect to time and primes denote differentiation with respect to $u$. Following steps similar to those in Sec. III we then find

$$
\ddot{u}=\frac{-\dot{u}^{2}\left(x^{\prime} x^{\prime \prime}+y^{\prime} y^{\prime \prime}\right)-g y^{\prime}}{\left(x^{\prime}\right)^{2}+\left(y^{\prime}\right)^{2}} .
$$

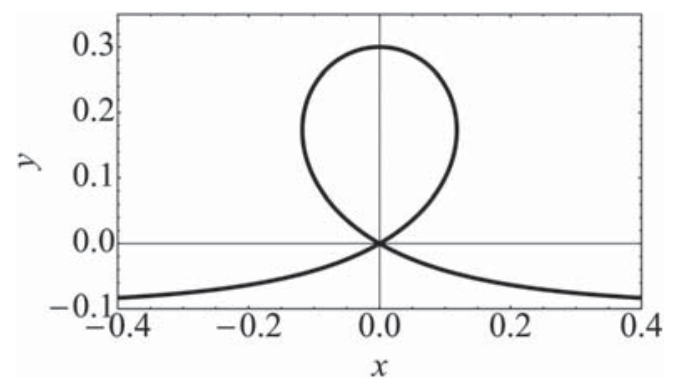

Fig. 8. The Trisectrix of MacLauren, an example of a multi-valued function resembling a "loop-the-loop" roller coaster track. This curve is described by Eqs. (13) and (14) and is plotted using $b=0.1 \mathrm{~m}$ and $-5 \leq u \leq 5$.

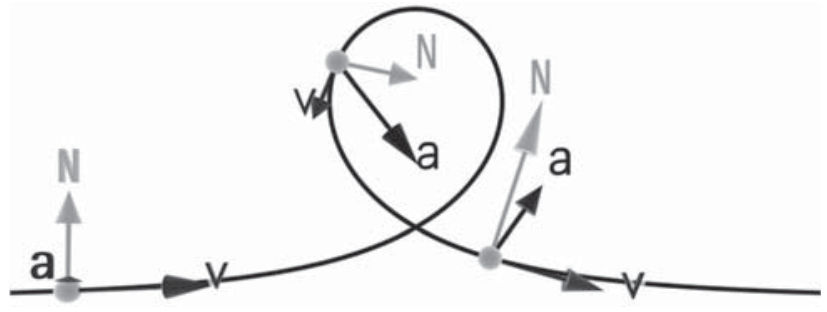

Fig. 9. Normal force $(\vec{N})$, velocity $(\vec{v})$, and acceleration $(\vec{a})$ vectors rendered at three different times for an object constrained to a loop-the-loop curve, modeled by a Trisectrix of MacLaurin. We use Eqs. (13) and (14) with parameters $b=3 \mathrm{~m}$ and $-7 \leq u \leq 7$. For integration of the object's motion we used $u_{0}=-7$ (see text), $v_{x 0}=17 \mathrm{~m} / \mathrm{s}$, and $m=1 \mathrm{~kg}$ (enhanced online) [URL: http://dx.doi.org/10.1119/1.4773561.2].

Equation (12) is a second-order nonlinear differential equation for the parameter $u$. If we can solve this equation for $u(t)$ then we can use Eqs. (8)-(11) to find the basic kinematic variables. All that remains is to set the initial conditions and use a numerical integration algorithm to find stepwise values for $u$. Some careful consideration is necessary as we need the initial values of $u(0)=u_{0}$ and $\dot{u}(0)=\dot{u}_{0}$. These initial values can be determined from the initial position and velocity in one of the coordinate directions; the initial conditions in the other coordinate can then be determined using Eqs. (8) and (9).

\section{EXAMPLE: THE TRISECTRIX OF MACLAURIN}

As an example of this generalized treatment, we parameterize a loop-the-loop curve by an adaptation of the Trisectrix of MacLaurin ${ }^{18}$ where

$$
x(u)=\frac{b u\left(u^{2}-3\right)}{u^{2}+1}
$$

and

$$
y(u)=\frac{-b\left(u^{2}-3\right)}{u^{2}+1} .
$$

This curve is shown in Fig. 8 for $b=0.1 \mathrm{~m}$ and $-5 \leq u \leq 5$.

Derivation of the analytic derivatives of Eqs. (13) and (14) for use in Eq. (12) are straightforward and will not be presented here. In computing the trajectory of an object constrained to this curve, $u_{0}$ is first selected (e.g., $u_{0}=-7$ ) so the object starts somewhere along the leftmost portion of the curve shown in Fig. 8. The choice of $\dot{u}_{0}$ is made more intuitive by first selecting a desired $v_{x 0}$ and then using Eq. (8) to find $\dot{u}_{0}=v_{x 0} / x_{0}^{\prime}$. [The same could be done using $v_{y}$ and Eq. (9).] From step $n$, we can find $\dot{u}$ at step $n+1$ using simple Euler steps, by first evaluating $\ddot{u}_{n}$ with $u_{n}$, then finding $\dot{u}_{n+1}$ $=\dot{u}_{n}+\ddot{u}_{n} \Delta t$, and $u_{n+1}=u_{n}+\dot{u}_{n} \Delta t$. With stepwise knowledge of $u, \dot{u}$, and $\ddot{u}$, the basic kinematic variables can be found using Eqs. (8)-(11). From these, other desired physical quantities are computable. An instantaneous look at the normal force, velocity, and acceleration vectors is shown in Fig. 9 at three different times. A sample animation of an object traversing the Trisectrix is available as supplementary material. ${ }^{12}$

\section{CONCLUSIONS}

Instructors wanting to incorporate computation into their introductory physics curriculum will find a need for numerical 
problems in addition to the analytical problems presented in standard university physics texts. This work presents the ideas needed to numerically model the common problem of an object moving along a sloped wire.

Our analysis has resulted in a generalized numerical approach to finding the piecewise trajectory of an object constrained to an arbitrary mathematical curve using a direct application of Newton's second law. Initially, we found a result for the case of the wire being described by a singlevalued function, appropriate for study in introductory mechanics for students with knowledge of basic calculus. This idea was extended to include parameterization of the curve, relaxing the requirement of the curve being single valued. In either case, the problem is that of solving a (nonlinear) second-order differential equation. We have done so using a simple Euler method and produced animations of the resulting motion.

From our experience, this approach is adaptable for lessons in an introductory physics course. The ideas here combine physics, calculus, and computational work. The technique allows for the computation of kinematic variables, the meaning of which should be familiar to students, which can then be used to compute other physical properties of the object as it traverses the wire. Although primarily used when discussing forces, the ideas here are used later when the potential and kinetic energies of the object are computed and illustrated in animations using "energy bar charts." 19 These ideas are used again in rotational kinematics where a round object is made to roll without slipping along a curve. The computational aspects demonstrate a continual reuse of past work with only small modifications needed to study new concepts. Lastly, in a course final project, a student may find or create a function of their own choosing for which to animate the traversal of an object. As a class project, one of our students animated an object oscillating on a parabolic wire, akin to a "skateboarder in a half-pipe.",20

\section{ADDITIONAL PROBLEMS}

The following ideas arose as this work was developed but were not suitable for assignments in an introductory physics course. Problems 1-4 can serve as analytical exercises to review and solidify the theory presented. Problems 5-10 can serve as student capstone projects.

1. Verify that the equations in Sec. III deliver the familiar results for an object on a rigid, straight wire inclined at an angle $\theta$ relative to the horizontal.

2. Starting with Eq. (3), complete the steps leading to Eqs. (4) and (5).

3. Verify that the result found in Ref. 11 is the same as Eq. (6).

4. In extending the results of Sec. VI, use $\vec{r}=x(u) \hat{i}+y(u) \hat{j}$ and verify that

$$
\vec{v}=\frac{d \vec{r}}{d t}=\frac{d x}{d u} \frac{d u}{d t} \hat{i}+\frac{d y}{d u} \frac{d u}{d t} \hat{j}
$$

and

$$
v^{2}=\left[\left(\frac{d x}{d u}\right)^{2}+\left(\frac{d y}{d u}\right)^{2}\right]\left(\frac{d u}{d t}\right)^{2},
$$

which are useful, if awkward, expressions when computing the momentum $(m \vec{v})$ or kinetic energy $\left(m v^{2} / 2\right)$.
5. Extending the results of Sec. V A for the " $1+$ tanh" function, let the initial speed vary and make a plot of the maximum of the normal force (magnitude) as a function of the initial speed.

6. For an object with a large initial speed sliding along the " $1+$ tanh" function, develop a computer animation that monitors the magnitude of the normal force to see if it passes to within some (small) $\epsilon$ of zero. This would correspond to the object on the verge of leaving the wire. At this instant (and thereafter) allow the object to be in free fall.

7. Modify the previous problem and find the intersection point between the free fall trajectory and the original curve and allow the object to rejoin the curve and continue on in constrained motion. What ramifications does rejoining the curve after free fall have on conservation of mechanical energy?

8. Using the approach described in Sec. VI, produce an animation of an object traversing the curve shown in Fig. 9. To use these results, you need to find the first and second derivatives of Eqs. (13) and (14). For initial conditions, we used the numbers given in the caption of Fig. 9. Experiment with $v_{x 0}$ and find cases for which the object is just able to traverse the loop. Compare with the result expected from energy conservation.

9. With the center-of-mass velocity $\vec{v}$ known at each instant as an object moves, it will roll without slipping if $v=R \omega$, where $R$ is the radius of the object and $\omega$ is the rotational speed. Using your chosen software package, animate an object rolling as it moves along an arbitrary curve. Carefully determine the instantaneous $\vec{\omega}$ and $\vec{\alpha}$ vectors and, for illustrative purposes, attach their tails to the center of rotation of the object. You may need to adjust your viewing perspective in order to see them.

10. Depending on the capabilities of your graphics software, the view point for the scene may be set to correspond to the instantaneous position and direction-of-travel of the object. Note that the instantaneous direction-of-travel is simply the velocity vector. This allows one to "ride along" with the object as it traverses the chosen path. Create such an animation. This may require adding unrelated background objects, such as a horizon, that the viewer may use to orient themselves as the animation proceeds.

\section{ACKNOWLEDGMENTS}

The authors thank R. Echols, T. Gutierrez, and K. Saunders for helpful discussions and T.B.'s introductory physics students, particularly T. W. Weber, during the 2008-2010 terms for their enthusiasm. The authors also thank an anonymous reviewer for efforts in implementing our theory, and encouraging us to further address numerical errors inherent in the simple numerical techniques used in this work.

\footnotetext{
${ }^{\text {a)} E l e c t r o n i c ~ m a i l: ~ t b e n s k y @ c a l p o l y . e d u ~}$

b)Electronic mail: mmoelter@calpoly.edu

${ }^{1}$ N. Chonacky and D. Winch, "Integrating computation into the undergraduate curriculum: A vision and guidelines for future developments," Am. J. Phys. 76, 327-333 (2008).

${ }^{2}$ R. W. Chabay and B. A. Sherwood, "Modern mechanics," Am. J. Phys. 72, 439-445 (2004).

${ }^{3}$ R. W. Chabay and B. A. Sherwood, Matter and Interactions I: Modern Mechanics (J. Wiley \& Sons, New Jersey, 2007).

${ }^{4}$ R. W. Chabay and B. A. Sherwood, "Computational physics in the introductory calculus-based course,” Am. J. Phys. 76, 307-313 (2008).
} 
${ }^{5}$ D. Scherer, P. Dubois, and B. Sherwood, "VPython: 3D interactive scientific graphics for students," Comput. Sci. Eng. 2, 56-62 (2000).

${ }^{6}$ T. J. Bensky, "Illustrating physics with ray-traced computer graphics," Phys. Teach. 44, 369-373 (2006).

${ }^{7}$ R. D. Knight, Physics for Scientists and Engineers, 2nd ed. (Pearson, San Francisco, 2004).

${ }^{8} \mathrm{We}$ emphasize our pedagogical needs are for introducing computer animation and programming into a introductory-level physics course. Other difficulties include objects that experience abrupt changes in net force (e.g., an object sliding off of a table or encountering friction), drag forces dependent on velocity, collision detection and scattering between two objects, modeling of ropes and pulleys, demonstrating convincing energy conservation with a simple Euler algorithm, illustrating rotational kinematics, and limits of motion.

${ }^{9}$ John Taylor, Classical Mechanics (University Basic Books, Sausalito, CA, 2000)

${ }^{10}$ D. A. Wells, Lagrangian Dynamics (McGraw-Hill, New York, 1967), p. 44.

${ }^{11}$ The Lagrangian approach is as follows. We start with kinetic and potential energies of $T=m\left(\dot{x}^{2}+\dot{y}^{2}\right) / 2$ and $V=m g y$, where $y$ is the object's height. Applying the Euler-Lagrange equation to the Lagrangian $L=T-V$, we obtain $\ddot{x}\left(1+y^{\prime 2}\right)+\dot{x}^{2} y^{\prime} y^{\prime \prime}+g y^{\prime}=0$, where $y=f(x)$ is the functional form of the wire curve. Here, primes (dots) indicate derivatives with respect to $x$ (time). This result is essentially our central result given by Eq. (6), but this approach is not appropriate for introductory physics.

${ }^{12}$ See http://dx.doi.org/10.1119/1.4773561 for some videos, an Easy Java Simulation, and our curricular materials.
${ }^{13} \mathrm{~A}$ second-order Runge-Kutta algorithm results in an essentially constant energy, to within the precision of the line thickness on the graph.

${ }^{14}$ Wolfgang Christian and Jan Tobochnik, "Augmenting AJP articles with computer simulations,” Am. J. Phys. 78, 885 (2010).

${ }^{15}$ Wolfgang Christian and Francisco Esquembre, "Modeling Physics with Easy Java Simulations," Phys. Teach. 45, 475-480 (2007). Our EJS version is available as supplementary material (see Ref. 12) or by email request to $\mathrm{T}$. Bensky.

${ }^{16} \mathrm{As}$ an example, imagine an object at the top of a hemisphere as in Problem \#16 (p. 301) of Ref. 7. The question asks at what position the object will leave the hemisphere as it slides down. This is an excellent problem to test the correspondence between the analytical and numerical solutions to the problem. The numerical solution is particularly insightful, forcing the student to consider how to examine the normal force, with the need to watch for it to become "zero" (i.e., sufficiently small).

${ }^{17}$ Efforts to integrate computers into introductory physics are worthwhile but difficult. We found almost no lesson-by-lesson guidance for doing so at this level. Decades of introductory physics-textbook authorship continues to be inconsistent with computational work, despite the dominance of computers in science. Our curricular materials, which covers introductory mechanics, including approximately 75 computational-based physics problems, are available as supplementary material (see Ref. 12).

${ }^{18}$ J. D. Lawrence, A Catalog of Special Plane Curves (Dover, San Francisco, 1972).

${ }^{19}$ For example, see Ref. 7, p. 273.

${ }^{20} \mathrm{~A}$ greatly embellished video version of a particle on a parabolic track can be viewed online or downloaded as supplementary material (see Ref. 12).

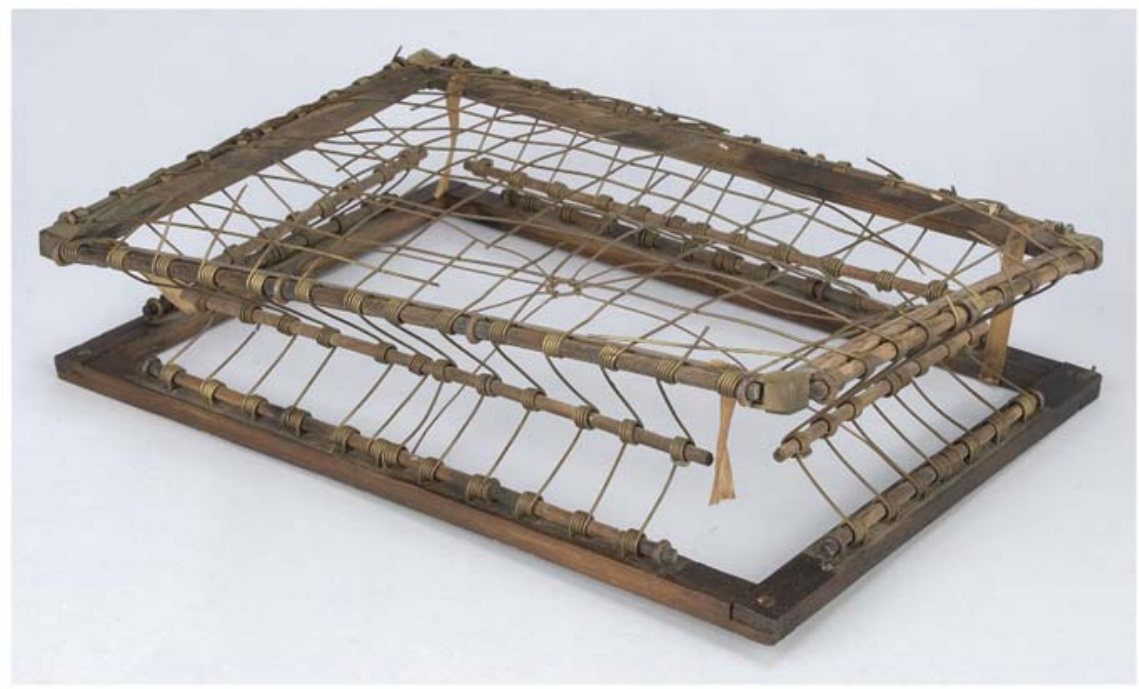

Model of a spring bed bottom

From 1790 until 1880 inventors were required to submit a model of their inventions along with their application to the U. S. Patent Office. This model, submitted by William W. Hawk in 1872, consists of two rectangular wooden frames on top of one another. The upper frame has four brass corners. Both frames are equipped with wooden rods on which wires are twisted around. The wires fixed on the top frame (several are broken) crisscross it in such a way that they form a dome-shaped square pattern. In between the two frames are the same wooden rods with twisted wires, giving the overall structure its springing quality. Finally, four pieces of cloth link the two frames together, one at each corner. The chief claim of this invention is that the structure is so designed as to entirely prevent sagging in the center, and that it will be equally elastic for a large range of weights placed on it.

This model is a part of the Susan Mae Eichelberg (Glendening/professional name, glendeningsusan@hotmail.com) collection, which includes over 400 patent models of historical significance. Thispatent model collection is one of the exhibits at The Riverbank Historic House Museum in Cornwall on Hudson, New York. The images and this description are courtesy of the Collection of Historical Scientific Instruments, Department of the History of Science, President and Fellows of Harvard College, where these patent models were exhibited in 2009. See patentmodelmuseum.org and riverbankhistorichousemuseum.org. 